\title{
A Lecture-Based Instructional System to Promote Student Agency
}

\author{
Dr. Matthew Merritt ${ }^{1}$, Dr. Athipat Cleesuntorn ${ }^{2}$, Dr. Laura Brahmakasikara ${ }^{3}$ \\ Assumption University of Thailand
}

\begin{abstract}
This research study was conducted in 2018 and resulted in an instructional system designed to support university instructors that seek to promote student agency in lecture-based learning environments. The objective of the study was to design and test an instructional system that supplements the traditional lecture and provides opportunities for the development of agentic engagement. In support of the instructional system design, the study examined ways in which university undergraduates used a digital backchannel, determined if using a digital backchannel affected agentic engagement, and identified the features of a digital backchannel that influenced student agency. The study employed a mixed methodology design using a questionnaire to collect quantitative student profile data and phenomenography to conduct a qualitative inquiry into participants' experience. The population for this study consisted of undergraduates at a private, International university located outside of Bangkok, Thailand. A total of 171 participants took part in this study, with ten students selected for a focus group through a non-probability, purposive sampling. Overall, the study found that a lecturing system that employs the strategic use of a digital backchannel can promote student agentic engagement. Student agency and instructor effectiveness were both positively influenced through the employment of an instructional system.
\end{abstract}

Keywords: agentic engagement; digital backchannel; instruction; lecture-based learning; technology 